\title{
Lernen eine Chance geben
}

_ From the mentor of the center to the guide on the side. Dieser Satz prägte sich mir im Zusammenhang mit Problemorientiertem Lernen ein. Lehrende sollen sich zurücknehmen, um ihren Schülern die Chance zu geben, lernen zu lernen. Sinngemäß heißt das: nicht predigen, sondern beratend zur Seite stehen. Der Nürnberger Trichter funktioniert sowieso nicht. Tobias Erhardt beschreibt das eindrücklich und nennt die Irrtümer, denen Lehrende immer wieder unterliegen (๑ S. 41). Beim Lesen des Artikels dachte ich übrigens: Sind wir nicht alle ein bisschen Markus? Schauen Sie mal, wie es Ihnen geht.

_ Die Botschaft vom Guide on the Side richtet sich auch an Therapeuten. Behandlungen beinhalten immer wieder Lehr-Lern-Situationen. Lehren wir dabei unsere Patienten, sich selbst zu helfen? Hand aufs Herz, die Patientenschulung ist sicher ausbaufähig. Und Zeitmangel spricht dafür und nicht dagegen. Das folgende Beispiel zeigt, dass selbst üben nützt.

_ Neulich erzählte mir der Kollege Florian Schneider von einer Studie, an der er beteiligt war. Der Ulmer Unfallchirurg Gert Krischak brachte das Ergebnis der Studie so auf den Punkt: Alleine üben macht Patienten mit distaler Radiusfraktur fitter! Eine Patientengruppe übte mithilfe eines von Physiotherapeuten erstellten Übungsheftes nach einmaliger Anleitung sechs Wochen lang selbstständig. Die andere Gruppe bekam zweimal wöchentlich Physiotherapie und wurde von den Therapeuten zu Heimübungen angehalten. Nach sechs Wochen verfügten die selbstständig Übenden über eine höhere Griffkraft und geringere Funktionsbeeinträchtigungen des Handgelenks. Die Verantwortung motivierte die Patienten offensichtlich. Das Studienergebnis hingegen widersprach den Erwartungen der Therapeuten, es verblüffte sie.

- Wenn Therapeuten oder Dozenten ihr Bestes geben, unter vollem Einsatz virtuoser Fingerfertigkeit behandeln oder dank nächtelang erstellter PowerPoint-Präsentationen detailreiche Vorträge zur Wissensvermehrung der Schüler halten, ist das sicher gut gemeint. Aber Sie wissen: das Gegenteil von gut gemeint ist gut.

Grüße vom Spielfeldrand und ... viel Spaß bei der WM

Ihre

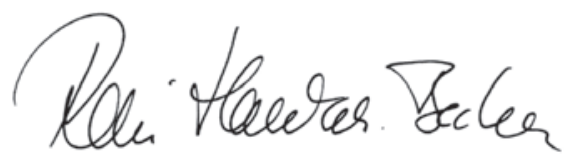

PS: Wir starten eine neue Reihe: Red Flags erkennen. Empfehlenswert!

\section{PHYSIOBONUS}

physiobonus heißen die Gewinnspiele in physiopraxis.

Wer teilnehmen möchte, nutzt das Formular unter www.thieme.de/ physioonline in der Rubrik physioexklusiv. Und das gibt es in dieser Ausgabe zu gewinnen:

Kursplatz

1 Seminar für das

Lehrerkollegium

Seite 42

1 Kurs „Herz-Kreislauf-

Trainer - Indoor“

Seite 62

Bücher

3-mal „Update

Physiotherapie“

Seite 22

3-mal „Unser Leben unsere Krankheit“

Seite 27

2-mal „Zur Optimierbarkeit der Klaviertechnik“

Seite 33

3-mal „Methoden-

Training“

Seite 42 